\title{
Ausencia de malaria asintomática en escolares de Quibdó, Chocó
}

\begin{abstract}
Lyda Osorio ${ }^{1,2}$, Jim Todd ${ }^{2}$, David Bradley ${ }^{2}$
${ }^{1}$ Centro Internacional de Entrenamiento e Investigaciones Médicas, CIDEIM, Cali Colombia.

${ }^{2}$ London School of Hygiene and Tropical Medicine, London, United Kingdom.

La malaria asintomática es considerada común en zonas de alta transmisión en África y prácticamente ausente en zonas de baja transmisión en Latinoamérica. Sin embargo, algunos reportes sugieren que el hallazgo de casos asintomáticos en zonas de alta y moderada transmisión en Latinoamérica es relativamente frecuente. La malaria asintomática tiene consecuencias graves no sólo para el individuo portador de parásitos, sino también para la comunidad donde vive. Los individuos crónicamente infectados se constituyen en un reservorio de la enfermedad que es difícil de identificar por medio de la vigilancia rutinaria de los programas de control.

En el marco de un estudio de la epidemiología de la malaria en Quibdó, Chocó, se estimó la prevalencia de malaria asintomática en escolares. El objetivo fue establecer si los individuos con infección asintomática podrían constituir un reservorio importante de infección, capaz de mantener la transmisión local en esta ciudad.

Se seleccionaron 255 estudiantes de cinco escuelas de Quibdó, de los cuales, 223 se incluyeron en el análisis. Algunos niños refirieron cefalea (34\%), tos (32\%) y diarrea (9\%). Todos los resultados de gota gruesa fueron negativos. Además, el análisis por inmunofluorescencia indirecta de anticuerpos contra Plasmodium en una submuestra de 25 niños fue negativo. La prevalencia estimada de malaria asintomática en los escolares de Quibdó es, entonces, de 0\% (IC95\%: 0-1,4). La presencia de malaria asintomática en adultos no se puede descartar aunque es probable que, si existe, sea en una proporción muy baja.
\end{abstract}

Palabras clave: malaria asintomática, Colombia, Chocó

\section{Absence of asymptomatic malaria in schoolchildren of Quibdó, Chocó}

Asymptomatic malaria is characteristic of high intensity transmission areas in Africa but unusual in low transmission areas in Latin America. Nevertheless, asymptomatic malaria has been reported to be frequent in areas in Latin America with high and moderate intensity of transmission. Asymptomatic malaria can affect both individuals who carry parasites and are cryptic carrier reservoirs for the community. Individuals chronically infected with malaria parasites are usually unidentifiable by most malaria control programmes.

In order to identify whether asymptomatic individuals harboring malaria parasites are an important reservoir of infection in Quibdó, Chocó, the prevalence of asymptomatic malaria was assessed in schoolchildren. This study was part of a major study of the epidemiology of malaria in Quibdó.

A total of 255 children from 5 schools were examined, of which 223 were included in the analysis. Children reported headache (34\%), cough (32\%), and diarrhoea (9\%). None of the children presented a positive thick smear. In addition, IFA tests in a subsample of 25 children were negative. By these criteria, the prevalence of asymptomatic malaria in Quibdó schoolchildren is $0 \%(95 \%$ C.I.: $0.0-1.4)$. Although asymptomatic malaria in adults possibly occurs, a very low prevalence is predicted.

Key words: asymptomatic malaria, Colombia, Chocó 
La presencia de síntomas típicos de malaria, como fiebre, escalofríos, sudoración y otros (cefalea, náuseas y malestar general) normalmente depende de la presencia de formas asexuales de Plasmodium en la sangre de los individuos infectados. Sin embargo, hay situaciones en las cuales la presencia de formas asexuales del parásito no se asocia con malaria clínica, por ejemplo, cuando el nivel de parasitemia está por debajo del umbral en el cual un individuo desarrolla síntomas (umbral pirogénico) o cuando el sistema immune mantiene los niveles de parasitemia bajos durante periodos largos y, así, al individuo libre de síntomas (1). Además, la malaria asintomática se puede observar como resultado de la administración intermitente de niveles subterapéuticos de antimaláricos (2).

En África es común observar recién nacidos y adultos que no desarrollan síntomas en el curso de una infección malárica. De hecho, a pesar de que la mayoría de la población sea portadora del parásito, las manifestaciones clínicas de la enfermedad y sus complicaciones se presentan principalmente en los niños (3).

Aunque los mecanismos que explican esta 'inmunidad clínica' no están totalmente esclarecidos, en recién nacidos, la ausencia de síntomas en presencia de infección malárica se ha asociado con la transferencia de anticuerpos maternos, baja ingestión de ácido p-aminobenzoico (PABA) durante la lactancia y presencia de hemoglobina fetal ( $\mathrm{Hb} \mathrm{F}$ ) (4). En adolescentes y adultos, la ausencia de síntomas se ha asociado con respuesta de anticuerpos contra moléculas expresadas en la superficie y fuera de ella de los estadios asexuales del parásito (5).

La prevalencia de malaria asintomática, así como la edad a la cual se desarrolla algún grado de protección contra los síntomas de la enfermedad, varía con la intensidad de la transmisión de malaria. En áreas donde la transmisión es alta (la población está expuesta a varias infecciones por

Correspondencia:

Lyda Osorio, CIDEIM, Avenida 1 Norte \# 3-03, Cali, Colombia Teléfono: (572) 668 2164; fax: (572) 6672989

lydaosorio@cideim.org.co

Recibido: 09/06/03; aceptado: 12/12/03 año), los síntomas y las complicaciones de la infección malárica son más frecuentes en niños menores de 5 años que en adolescentes y adultos. Con la disminución en el nivel de transmisión, la edad a la cual se adquiere 'protección' contra las manifestaciones clínicas de la infección malárica se va retardando hasta que, en zonas de muy baja intensidad, prácticamente todos (niños y adultos) tienen un riesgo similar de enfermarse (3). Además de los factores mencionados anteriormente que confieren cierto grado de protección a los recién nacidos, la relación entre edad, morbilidad e intensidad de la transmisión se cree que es el resultado de la inmunidad adquirida a través de la exposición a un número acumulado de infecciones en el tiempo (6). Esta 'inmunidad clínica' se ha observado que es específica de especie, estadio del parásito y cepa (4), lo cual sugiere, en vista de la alta diversidad antigénica de los Plasmodia, que para adquirir cierto grado de protección contra las manifestaciones clínicas de la malaria se requeriría experimentar infecciones con una amplia gama de Plasmodia. En zonas de baja transmisión como Colombia, donde un individuo presenta 1 o menos episodios de malaria por año, la malaria asintomática parece ser poco común. Sin embargo, Gupta et al., usando modelos matemáticos, sugieren que la protección contra las formas graves no cerebrales de la enfermedad se alcanza después de pocas infecciones (7). A pesar de esto, el grado de exposición a malaria que se requiere para ganar cierto grado de inmunidad contra la forma no complicada de la infección es aún controvertido.

La malaria asintomática afecta tanto al individuo portador de parásitos como a la comunidad en la que vive el individuo infectado. En individuos crónicamente infectados con Plasmodium, se puede observar esplenomegalia, anemia y síndrome nefrótico (8). En embarazadas, la malaria asintomática puede causar anemia e infección de la placenta, lo cual puede conducir a parto prematuro y bajo peso al nacer $(9,10)$.

Para la comunidad, la consecuencia más evidente de la malaria asintomática es que los individuos infectados no reciben tratamiento, los parásitos (incluso, gametocitos) permanecen en la 
circulación periférica y, por ende, se convierten en un reservorio de la infección para los mosquitos. Varios experimentos han mostrado que los mosquitos vectores de malaria pueden adquirir parásitos de individuos infectados tanto sintomáticos como asintomáticos (11-14). Los individuos con gametocitos observables por microscopía son los más infecciosos, aunque aquéllos con gametocitos por debajo del nivel de detección por microscopía de luz, también producen infecciones en los mosquitos (15). Esta observación es importante, puesto que los individuos asintomáticos pueden tener parásitos en densidades muy bajas (detectables por PCR) durante varios meses $(16,17)$.

En el marco de un estudio sobre la epidemiología de la malaria en Quibdó, Chocó, evaluamos la prevalencia de infección asintomática con el fin de establecer si los portadores asintomáticos de malaria podrían ser una fuente importante de parásitos, capaz de mantener la transmisión local de malaria en esta ciudad.

\section{Materiales y métodos}

\section{Población de estudio}

Quibdó reporta casos de malaria todo el año, con picos en los meses relativamente más secos. En los últimos 10 años, la incidencia anual de malaria ha variado entre 4,02/1.000 hab en 1994 y más de 150/1.000 hab en 1998. Durante la realización del estudio (2001), se observó un incremento en la incidencia de malaria $(37,23 / 1.000$ hab) con respecto al 2000 (12,32/1.000 hab). Se reporta la presencia de Plasmodium falciparum y de Plasmodium vivax en la ciudad. Sin embargo, $P$. falciparum predomina con una proporción de $66 \%$, aproximadamente; cerca del $33 \%$ de los casos son debidos a $P$. vivax, y el $1 \%$ restante se clasifican como infecciones mixtas ( $P$. falciparum y $P$. vivax) (18). La malaria en Quibdó se puede clasificar como de baja intensidad de transmisión ya que, según la incidencia, en general, los habitantes experimentan menos de un episodio de malaria por año.

\section{Muestreo}

El estudio se realizó en escolares de Quibdó, dado que no se encontraba disponible un marco muestral (una lista de los habitantes de la ciudad) para hacer un muestreo simple al azar de la población. Para la selección de la población de estudio, se usó un muestreo por agregados en dos etapas. En la primera etapa, se tomó una muestra al azar de las escuelas de primaria de Quibdó y en la segunda etapa, una muestra al azar de los estudiantes de las escuelas seleccionadas. La lista de las escuelas tanto públicas como privadas se obtuvo en la Secretaría de Educación del departamento y se actualizó con los miembros de la comunidad hasta que se obtuvo un consenso de que estaba completa. Se encontró que faltaban 5 escuelas privadas que se adicionaron a la lista provista por la Secretaría de Educación. A cada escuela en la lista se le asignó un número, el cual se usó para seleccionar las escuelas con base en una tabla de números aleatorios (19). En vista de que las escuelas seleccionadas diferían en el número de estudiantes, el número de niños seleccionados por escuela se calculó con base en la técnica de muestreo con probabilidad proporcional al tamaño (20). De esta manera, cada niño tuvo la misma probabilidad de ser seleccionado.

\section{Procedimientos}

A los escolares seleccionados para el estudio se les interrogó sobre su estado de salud y, particularmente, por la presencia de cefalea, tos, diarrea y fiebre. A los padres de los niños seleccionados se les preguntó sobre la salud del niño en la semana previa a la entrevista; si los niños habían recibido antimaláricos o antibióticos con efecto antimalárico, como sulfas; si el niño había tenido malaria confirmada por microscopía en el último año, y si alguien en la casa había presentado malaria confirmada por microscopía en el último mes. Después de la entrevista, a cada niño se le practicó un examen clínico general que incluyó toma de la temperatura axilar, revisión de los órganos de los sentidos, detección de signos de anemia (palidez de conjuntivas) y de dificultad respiratoria. Adicionalmente, se investigó la presencia de hepatomegalia y esplenomegalia y se obtuvo una muestra de gota gruesa por punción digital para investigar la presencia de Plasmodium.

Para la toma de la temperatura, se usó un termómetro digital, el cual se calibró diariamente. 
La palpación de bazo se hizo con el niño de pie y con el examinador sentado en frente del niño, como describen Wernsdorfer y MacGregor (21).

La gota gruesa se coloreó con Giemsa y una sola persona leyó todas las láminas con un aumento de 1.000X en un microscopio de luz (Nikon Alphaphot YS2-H3). La gota gruesa se consideró negativa si no se encontraron parásitos en, al menos, 200 campos examinados. Un segundo lector, que no conocía los resultados del primer lector, leyó el $50 \%$ de las láminas. Los resultados del primer y segundo lector fueron idénticos.

Se obtuvo una muestra de sangre en papel filtro por punción digital en 220 niños. Veinticinco de estas muestras se seleccionaron al azar con base en una tabla de números aleatorios, para realizar pruebas de inmunofluorescencia indirecta (IFI) de anticuerpos contra Plasmodium. Las pruebas IFI se realizaron en el Laboratorio de Referencia de Malaria de la London School of Hygiene and Tropical Medicine (LSHTM);se usó como antígeno $P$. falciparum procedente de cultivos in vitro según la metodología descrita por Warrell y Gilles (22).

\section{Consideracion éticas}

Los padres de los niños seleccionados fueron invitados a la escuela, en donde el investigador principal explicó los objetivos y los procedimientos del estudio. Se obtuvo el consentimiento firmado de cada padre o del responsable de cada niño antes de cualquier procedimiento. Todos los resultados de los exámenes se entregaron a los padres y, en caso de detectarse alguna anormalidad en el examen médico, se dieron recomendaciones para que buscaran atención médica.

El estudio fue aprobado por los comités de ética del LSHTM y del Centro Internacional de Entrenamiento e Investigaciones Médicas (CIDEIM).

\section{Cálculo del tamaño de la muestra} $y$ análisis de los datos

El tamaño de la muestra se calculó en 250 niños con base en una prevalencia de malaria asintomática esperada de $3 \%$, un error estándar de $1,5 \%$, un intervalo de confianza del $95 \%$ ( 2 colas) y un efecto del diseño de 2. El número de escuelas que se debía incluir fue de 5 y se seleccionó con base en la fórmula para agregados de Bennett et al. (20).

Para la entrada de datos se usó el programa Epi Info 6.04d (CDC/WHO 2001) y Stata 7 (Stata Corporation 2001) para el análisis descriptivo. Se obtuvo la frecuencia de las características demográficas de los niños, su estado de salud y los resultados del examen físico. Se calculó la proporción de gotas gruesas positivas y el correspondiente intervalo de confianza del $95 \%$ a partir de la distribución de Poisson.

\section{Resultados}

En el estudio se incluyeron 255 niños: 50 de una escuela con 301 estudiantes, 37 de una con 190 estudiantes, 50 de una con 267 estudiantes, 66 de una con 375 estudiantes y 52 de una escuela con 275 estudiantes. Tres escuelas estaban localizadas en el centro de la ciudad y 2 en el área periurbana. A 32 niños que habían tomado medicamentos antimaláricos $(n=10)$ y que no estaban seguros si habían tomado o no $(n=22)$, se les leyó la gota gruesa pero fueron excluidos del análisis.

En total, 223 niños fueron incluidos en el análisis. El promedio de edad fue de 9 años (rango: 5 a 16). La distribución por sexo fue similar, con 105 (47\%) niños y 118 (53\%) niñas. En los exámenes de gota gruesa no se observaron Plasmodia en ninguno de los niños. Tampoco se encontraron niños con esplenomegalia en el examen clínico. Todas las 25 muestras que se examinaron para anticuerpos antiplasmodium fueron negativas.

Los niños refirieron síntomas como: cefalea, 76 (34\%); tos, $71(32 \%)$, y diarrea, $21(9 \%)$. Los padres de $29(13 \%)$ niños reportaron que sus hijos habían presentado fiebre en la semana previa a la entrevista. Sin embargo, un solo niño se encontró febril (temperatura $>37,5^{\circ} \mathrm{C}$ ) en el momento del examen clínico. Este niño tenía también hepatomegalia e ictericia.

Dado que ninguno de los 223 niños se encontró positivo para la infección por Plasmodium, la prevalencia estimada de malaria asintomática en escolares de Quibdó es de 0\% (IC95\%: 0-1,4). 


\section{Discusión}

La malaria asintomática se considera como una característica común en zonas de alta transmisión en África, pero inusual en zonas de baja transmisión en Latinoamérica. Los resultados del presente estudio sugieren que la malaria asintomática es infrecuente en Quibdó, la cual tiene una incidencia anual promedio de malaria de 38 casos $/ 1.000$ hab y se considera una zona de baja transmisión. Nuestros hallazgos son similares a los de Méndez et al., quien encontró una baja prevalencia de malaria asintomática $(1,4 \%$ $=19 / 1.380$ ) en otra zona urbana en la Costa Pacífica colombiana (Buenaventura) (23).

Sin embargo, otros estudios han demostrado que la prevalencia de malaria asintomática en la Costa Pacífica colombiana varía según la intensidad de la transmisión. Por ejemplo, en una zona cercana a Buenaventura, donde la transmisión de malaria es mayor que en Quibdó y Buenaventura, la prevalencia de malaria asintomática fue de 5,6\% (24), y en una zona cercana a esta última, donde el $20,4 \%$ de la población se encontró positiva para parásitos, la mayoría (>90\%) estaba asintomática (25). Así mismo, en otra región de Surámerica (Brasil) se encontró una prevalencia alta (14\%) de malaria asintomática (26). En todos estos estudios, la ausencia de síntomas en el momento del diagnóstico se podría explicar parcialmente por la adquisición de cierto grado de inmunidad en la población. Esta explicación es más probable en zonas con alta o moderada transmisión de malaria, donde las personas estarían expuestas a varias infecciones por año y eventualmente tendrían inmunidad adquirida. Pero en áreas donde la transmisión es de baja intensidad, es decir, donde en general las personas están expuestas a menos de una infección por año, las probabilidades de desarrollar inmunidad adquirida son menores y otros factores, como la detección precoz de la infección antes de que el individuo desarrolle los síntomas y la presencia de niveles subterapéuticos de medicamentos, podrían explicar la mayoría de los casos asintomáticos. Para este último, la historia de automedicación en el $28 \%$ de los individuos infectados, reportada en el estudio de Méndez et al. es un ejemplo (23).
Con el fin de establecer si la malaria asintomática es debida a la detección temprana de la infección, es necesario realizar estudios de seguimiento en los cuales se observa en el tiempo si los individuos infectados desarrollan síntomas. Prata et al. observaron durante 7 días a pacientes inicialmente asintomáticos y encontró que la mayoría (25/30) de ellos desarrolló síntomas y necesitó tratamiento. Cuatro pacientes habían tomado medicamentos antimaláricos, lo cual probablemente explicaba la ausencia inicial de síntomas; mientras que sólo un paciente, que había tenido múltiples episodios de malaria, permaneció asintomático (27). Este hallazgo sugiere que la malaria asintomática sí está presente en la región, pero que una 'verdadera' infección crónica es rara.

A pesar de lo anterior, la epidemiología de la malaria asintomática en zonas de alta transmisión en Latinoamérica refleja aquélla de zonas de alta transmisión en otros lugares. Recientemente, Alves et al. reportaron que individuos residentes en dos poblaciones en el Amazonas brasilero, que se encontraron infectados, permanecieron asintomáticos durante 2 meses de seguimiento (28). La prevalencia de malaria asintomática en estas poblaciones fue de $20 \%$ y $49,5 \%$, mientras que la incidencia total de malaria fue de más de 300 casos/1.000 hab entre 1998 y 1999. En este mismo estudio, Alves et al. reportaron que la ausencia de síntomas fue más frecuente en los habitantes nacidos en la región, adolescentes y adultos jóvenes, mientras que los inmigrantes y niños eran más propensos a presentar síntomas. La protección contra los síntomas de la enfermedad se adquirió en esta zona a partir de los 15 años (28). En Quibdó, la malaria en sus formas complicada y no complicada se observa tanto en adultos como en niños, lo cual indirectamente apoya los resultados del presente estudio. Sin embargo, puesto que el estudio se realizó en escolares, no se descarta la posibilidad de malaria asintomática en adultos, aunque ésta tendría probablemente una prevalencia muy baja. El estudio se realizó en escolares por la disponibilidad de realizar un muestreo al azar de esta población. Se han desarrollado métodos de muestreo que no requieren un marco muestral $(29,30)$, pero no se usaron porque su aplicación 
hubiese demandado recursos que no estaban disponibles para el presente estudio.

Una consideración adicional de la metodología empleada en el presente trabajo sería la potencial baja sensibilidad de la gota gruesa en niños con bajo nivel de parasitemia. En este caso se podrían utilizar pruebas con mayor sensibilidad como las basadas en la reacción en cadena de la polimerasa (PCR) (31). Sin embargo, debido al mayor costo de las pruebas de PCR se optó por realizar la prueba de IFI, la cual indica si el individuo ha estado en contacto con el parásito. Los resultados negativos de IFI en un subgrupo de escolares descartan la posibilidad de falsos negativos en la gota gruesa, puesto que los niños infectados hubiesen sido detectados por este método.

\section{Agradecimientos}

Agradecemos al personal de Dasalud-Chocó, a los directores, profesores, estudiantes y padres de familia de las escuelas seleccionadas, por su colaboración en la realización del presente trabajo. Nuestros agradecimientos al personal del Laboratorio de Referencia de Malaria en LSHTM por su ayuda con las pruebas de IFI. Este estudio fue financiado por WHO/TDR. Lyda Osorio recibe una beca-crédito de Colciencias.

\section{Referencias}

1. WHO. Terminology of malaria and of malaria eradication. Report of a drafting committee. Geneva: World Health Organization; 1963.

2. Rieckmann KH. Asymptomatic malaria. Lancet 1970;1: 82-3.

3. Molineaux L. The epidemiology of human malaria as an explanation of its distribution, including some implications for its control. En: Wernsdorfer WH, McGregor SI, editors. Malaria: principles and practice of malariology. London: Churchill Livingstone; 1988. p.968.

4. McGregor IA, Wilson RJM. Specific immunity: acquired in man. En: Wernsdorfer WH, McGregor SI, editors. Malaria: principles and practice of malariology. London: Churchill Livingstone; 1988. p.559-619.

5. Marsh K. Immunology of malaria. En: Warrell D, Gilles $\mathrm{H}$, editors. London: Arnold Publishers; 2002.

6. Baird JK. Age-dependent characteristics of protection vs. susceptibility to Plasmodium falciparum. Ann Trop Med Parasitol 1998;92:367-90.
7. Gupta S, Snow R, Donnelly C, Marsh K, Newbold C. Immunity to non-cerebral severe malaria is acquired after one or two infections. Nat Med 1999;5:340-3.

8. Greenwood BM. Asymptomatic malaria infections do they matter? Parasitol Today 1987;3:206-14.

9. McGregor IA. Thoughts on malaria in pregnancy with consideration of some factors which influence remedial strategies. Parassitologia 1987;29:153-63.

10. Okoko BJ, Ota MO, Yamuah LK, Idiong D, Mkpanam SN, Avieka A, Banya WA, Osinusi K. Influence of placental malaria infection on foetal outcome in the Gambia: twenty years after lan Mcgregor. J Health Popul Nutr 2002;20:4-11.

11. Boudin C, Olivier M, Molez JF, Chiron JP, Ambroise-Thomas P. High human malarial infectivity to laboratory-bred Anopheles gambiae in a village in Burkina Faso. Am J Trop Med Hyg 1993;48:700-6.

12. Tchuinkam $\mathbf{T}$, Mulder $B$, Dechering $K$, Stoffels $\mathbf{H}$, Verhave JP, Cot M, Carnevale P, Meuwissen JH, Robert V. Experimental infections of Anopheles gambiae with Plasmodium falciparum of naturally infected gametocyte carriers in Cameroon: factors influencing the infectivity to mosquitoes. Trop Med Parasitol 1993;44:271-6.

13. Lensen A, Mulder L, Tchuinkam T, Willemsen L, Eling W, Sauerwein R. Mechanisms that reduce transmission of Plasmodium falciparum malaria in semiimmune and nonimmune persons. J Infect Dis 1998; 177:1358-63.

14. Drakeley CJ, Secka I, Correa S, Greenwood BM, Targett GA. Host haematological factors influencing the transmission of Plasmodium falciparum gametocytes to Anopheles gambiae s.s. mosquitoes. Trop Med Int Health 1999;4:131-8.

15. Pethleart A. Importance of asymptomatic malaria and its infectivity to Anopheles mosquitoes in Mae Hong Son Province, Thailand. En: Department of Infectious and Tropical Diseases, University of London. London: London School of Hygiene and Tropical Medicine; 2002. p.245.

16. Gilles H. Tropical clinical epidemiology- 'a new name for an old art'. Trans R Soc Trop Med Hyg 1986;80:353-9.

17. Roper C, Elhassan IM, Hviid L, Giha H, Richardson W, Babiker H, Satti GM, Theander TG, Arnot DE. Detection of very low level Plasmodium falciparum infections using the nested polymerase chain reaction and a reassessment of the epidemiology of unstable malaria in Sudan. Am J Trop Med Hyg 1996;54:325-31.

18. Arriaga A. Estadísticas de malaria en Chocó. Quibdó: Departamento Administrativo de Salud (Dasalud, Chocó); 2000.

19. Kirkwood B. Essentials of medical statistics. London: Blackwell Science Ltd.; 1988. 
20. Bennett S, Woods T, Liyanage WM, Smith DL. A simplified general method for cluster-sample surveys of health in developing countries. World Health Stat $Q$ 1991;44:98-106.

21. Wernsdorfer WH, McGregor SI. Malaria. Principles and practice of malariology. First edition. London: Churchill Livingstone; London; 1988. p.1818.

22. Hommel M. Diagnostic methods in malaria. En: Warrell $\mathrm{D}$, Gilles $\mathrm{H}$, editors. Essential malariology. London: Arnold Publishers; 2002. p.51.

23. Méndez F, Carrasquilla G, Muñoz A. Risk factors associated with malaria infection in an urban setting. Trans R Soc Trop Med Hyg 2000;94:367-71.

24. González J, Olano V, Vergara J, Arévalo-Herrera M, Carrasquilla G, Herrera S, López J. Unstable, low-level transmission of malaria on the Colombian Pacific Coast. Ann Trop Med Parasitol 1997;91:349-58.

25. Gautret P, Barreto M, Méndez F, Zorrilla G, Carrasquilla G. High prevalence of malaria in a village of the Colombian Pacific coast. Mem Inst Oswaldo Cruz 1995;90:559-60.

26. Andrade A, Martelli C, Oliveira R, Arias J, Zicker F, Pang L. High prevalence of asymptomatic malaria in gold mining areas in Brazil. Clin Infect Dis 1995; 20:475.

27. Prata A, Urdaneta M, McGreevy P, Tada $\mathbf{M}$. Infrequency of asymptomatic malaria in an endemic area in Amazonas, Brazil. Rev Soc Bra Med Trop 1988;21: 51-4.

28. Alves F, Durlacher R, Menezes M, Krieger M, Pereira da Silva L, Camargo E. High prevalence of Plasmodium vivax and Plasmodium falciparum infections in native amazonian populations. Am J Trop Med Hyg 2002;66: 641-8.

29. Lemeshow S, Tserkovnyi AG, Tulloch JL, Dowd JE, Lwanga SK, Keja J. A computer simulation of the EPI survey strategy. Int J Epidemiol 1985;14:473-81.

30. Bennett S, Radalowicz A, Vella V, Tomkins A. A computer simulation of household sampling schemes for health surveys in developing countries. Int $J$ Epidemiol 1994;23:1282-91.

31. Snounou G, Viriyakosol S, Zhu XP, Jarra W, Pinheiro L, do Rosario VE, Thaithong S, Brown $\mathbf{K N}$. High sensitivity of detection of human malaria parasites by the use of nested polymerase chain reaction. Mol Biochem Parasitol 1993;61:315-20. 